Laurent, N., Lejard, C., Deltel, G. \& Bijl, P. (2013). Performance characterization of night vision equipment based on Triangle Orientation Discrimination (TOD) methodology. In Israel, K.R. \& Whelan, D.A. (Eds.), Infrared Imaging Systems : Design, Analysis, Modeling, and Testing XXIV. 2013 Defense Security+Sensing, Conference 8706, Baltimore, MD, USA (pp. 43). : SPIE doi:10.1117/12.2015382

\title{
Performance characterization of night vision equipment based on Triangle Orientation Discrimination (TOD) methodology
}

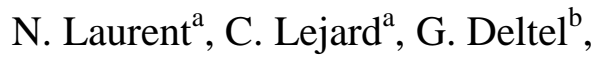 \\ P. Bijl ${ }^{\mathrm{c}}$
}

${ }^{\text {a }}$ PHOTONIS France SAS Avenue Roger Roncier 19600 Brive La Gaillarde France; email : n.laurent@fr.photonis.com

${ }^{\mathrm{b}}$ PHOTONIS Technologies SAS Axis Business Park - Bât E,18 avenue de Pythagore 33700 Mérignac France; email : g.deltel@photonis.com

${ }^{\mathrm{c}}$ TNO Behavioural and Societal Sciences, Kampweg 5, 3769 DE, The Netherlands; email: piet.bij1@tno.nl

\begin{abstract}
Night vision equipment is crucial in order to accomplish supremacy and safety of the troops on the battlefield. Evidently, system integrators, MODs and end-users need access to reliable quantitative characterization of the expected field performance when using night vision equipment. The Image Intensifier tube is one of the most important engines driving the performance for night vision equipment. As a major tube manufacturer, PHOTONIS has investigated the link between its products physical design parameters and the actual end-user field performance. The developments include 1) an end-to-end performance measurement method and test facility, 2) an image-based night vision simulation and 3) a range estimation model. The purpose is twofold: i) being able to support the need of the integrators and end users, and ii) further systematic improvement of night vision equipment design. For the end-to-end test, PHOTONIS and TNO cooperated in the implementation of the TOD (Triangle Orientation Discrimination) test for night vision equipment. This test provides a clear and rigorous ranking of the products with respect to their target acquisition performance level. With respect to the image-based simulation, PHOTONIS performs physical and performance comparisons between artificial and real imagery, promising exciting further development of a model based on the merging of the different approaches of night vision evaluation and modelling. In this paper, we present the PHOTONIS night vision test laboratory, provide TOD results for a set of night vision devices and show range prediction examples.
\end{abstract}

Keywords: Field performance, TOD test, Image Intensifier Tube, Night Vision Equipment, ICMOS

\section{INTRODUCTION}

As an imaging sensor manufacturer, the Photonis company has a continuous interest in foreseeing the night vision devices field performance embedding state-of-the-art Image Intensifier $\left(\mathrm{I}^{2}\right)$ tubes or new digital sensors solutions (ICMOS, Low Noise CMOS, EBCMOS). The major concern is to focus on the end user: what is the link between sensor performance increase and soldier task improvement. A task to predict sensor field performance is to collect end-to-end performance data such as a set of Minimum Resolvable Contrast (MRC) curves $^{3}$. In these tests, the observer task is to determine the lowest contrast at which a test pattern of certain size or spatial frequency can be resolved with the equipment under test. The test is repeated for a range of test pattern sizes and - in the case of night vision devices- for a range of light levels. The curves can be used to predict range performance with the sensor under operational circumstances. The test needs to be performed under well-defined and controlled conditions and special care needs to be taken to minimize the effect of subjective observer criteria. For these reasons, a rigorous bias -free protocol with humanin-the-loop is needed.

At Photonis, a number of range tests were implemented in the past few years in order to build the company own range model. The USAF target test ${ }^{4}$ is the most commonly used. The test is very simple, but it has proved to be not capable to demonstrate current improvements of recent $\mathrm{I}^{2}$ at Photonis. Therefore Photonis has decided the experimentation and the implementation of a "forced-choice" test, the Triangle Orientation Discrimination (TOD) ${ }^{5}$ method, which produces satisfactory results, very sensitive to $\mathrm{I}^{2}$ parameters change.

In the goal of having a reliable range performance test, Photonis put in place a test setup with a TOD test in collaboration with TNO institute. The goal is to assess the low light performance of different night vision devices, 
including both conventional $\mathrm{I}^{2}$ and CMOS based Photonis products. After consulting TNO in 2012 all recommendations were applied and these led to the tests and results described in the present paper.

The paper is organized as follows. In Section 2, a detailed description of the experimental setup is provided. This section also includes the sensors from two groups : three tubes from the standard Photonis group products portfolio and one ICMOS sample. This illustrates the ability of the TOD method to cover both conventional $\mathrm{I}^{2}$ and digital night vision devices. Section 3 shows the TOD measurement results for a broad range of night levels and all four sensor types. In Section 4 we discuss the results, illustrated with some range predicition examples and present the follow-up that will be conducted to further characterize all Photonis innovative night vision equipment.

\section{EXPERIMENTAL SET UP}

\subsection{Test devices}

\subsubsection{Image intensifiers $\left(\mathrm{I}^{2}\right)$}

Three different tubes that are representative for the PHOTONIS company portfolio were submitted to the test. Table 1 presents the main physical parameters defining the overall performance of these image intensifier tubes: Signal to Noise Ratio (SNR) and Limiting Resolution (LR). As defined in the MIL specifications, SNR quantifies the temporal noise of an image when the photocathode is illuminated by a light source at $108 \mu$ lux and at a color temperature of $2856+/-50^{\circ} \mathrm{K}$. SNR is measured over a maximum $200 \mu \mathrm{m}$ diameter spot, through a $10 \mathrm{~Hz}$ electronic bandwidth filter. The limiting resolution (LR) quantifies the maximum spatial frequency in $\mathrm{lp} / \mathrm{mm}$ seen by the observer when looking through a $10 \mathrm{x}$ magnifying ocular at a optimum input illumination (in practice several millilux). they. One can derive a Figure of Merit (FOM) that is equal to SNR times LR.

Table 1 : Signal to Noise Ratio (SNR) and Limiting Resolution (LR) of the three $\mathrm{I}^{2}$ used in the test

\begin{tabular}{|l|c|c|c|c|c|}
\hline Product & Specified SNR & Measured SNR & $\begin{array}{c}\text { Specified LR } \\
(\mathbf{l p / m m})\end{array}$ & $\begin{array}{c}\text { Measured LR } \\
(\mathbf{l p} / \mathbf{m m})\end{array}$ & $\begin{array}{c}\text { Figure Of } \\
\text { Merit (FOM) }\end{array}$ \\
\hline SuperGen $^{\circledR}$ & $18 \mathrm{~min}$ & 19 & $51 \mathrm{~min}$ & 54 & 1020 \\
\hline $\mathrm{XD}^{\mathrm{TM}}$ & $20 \mathrm{~min}$ & 22.3 & $60 \mathrm{~min}$ & 64 & 1430 \\
\hline $\mathrm{XR5}^{\mathrm{TM}}$ & $25 \mathrm{~min}$ & 26.7 & $64 \mathrm{~min}$ & 66 & 1760 \\
\hline
\end{tabular}

Each $\mathrm{I}^{2}$ was mounted in an identical monocular, having a typical FOV of $40^{\circ}$ and $\mathrm{f} / 1.2$ aperture . The tubes were set at a luminous gain of $13000 \mathrm{~cd} / \mathrm{m}^{2} / \mathrm{lux}$ and at a maximum output brightness of $6 \mathrm{~cd} / \mathrm{m}^{2}$. Maximum output brightness is then reached at a photocathode illumination of around $500 \mu$ lux.

\subsubsection{Intensified CMOS (ICMOS)}

The test set is completed by an ICMOS embedding a XD4 $4^{\mathrm{TM}}$ and a 1 " format 1.3 Mpixels CMOS sensor. $\mathrm{I}^{2}$ and CMOS are optically coupled with a optic fiber bundle. The resulting digital night vision (NV) sample serves for a preliminary comparison to the conventional $\mathrm{I}^{2}$ test set. The ICMOS was equipped with a $25 \mathrm{~mm}$ focal length lens, with $\mathrm{f} / 1.2$ Numerical Aperture (Lensagon CHS25095), thus introducing no bias in the comparison of standard and digital technologies (same FOV, same NA).

\subsection{Photonis test dark room}

In 2010, Photonis built a dark room for testing night vision equipment within a controlled environment. It is 4 meters wide and 13 meters long and fully dressed with black clothes in order to reach the full darkness. On one side of the room, observers can look through night vision devices and see a complete set of charts, placed at the other side of the room. Distance between sensor and targets can vary between 3 meters and 12 meters. Illumination is fixed thanks to a remote controll and periodic monitoring whether the ambient light (illumination and spectrum) is correct.

Illumination at five night level ranges is provided by 5 tungsten halogen light sources, at a fixed color temperature of $2856+/-50^{\circ} \mathrm{K}$, diaphragmed such that they project the desired illumination level on the targets with a good uniformity. Sources are placed at $9.50 \mathrm{~m}$ distance from the test targets described in section 2.3 . The five ranges and the illumination levels on the test charts used during the test are provided in Table 2. 
Table 2 : Moon phases of the five standard night levels illumination ranges and real illumination levels used in the tests

\begin{tabular}{|c|c|c|c|c|c|}
\hline Designation & Night Level 1 & Night Level 2 & Night Level 3 & Night Level 4 & Night Level 5 \\
\hline Light source & Full moon & Half moon & Partial moon & Clear Starlight & Overcast Starlight \\
\hline IlluminationRange & 40-1000 mlux & 10-40 mlux & 2-10 mlux & $0.7-2$ mlux & $0-0.7$ mlux \\
\hline $\begin{array}{l}\text { Test Room } \\
\text { Illumination }\end{array}$ & 100 mlux & 15 mlux & 4.3 mlux & 0.9 mlux & 0.4 mlux \\
\hline
\end{tabular}

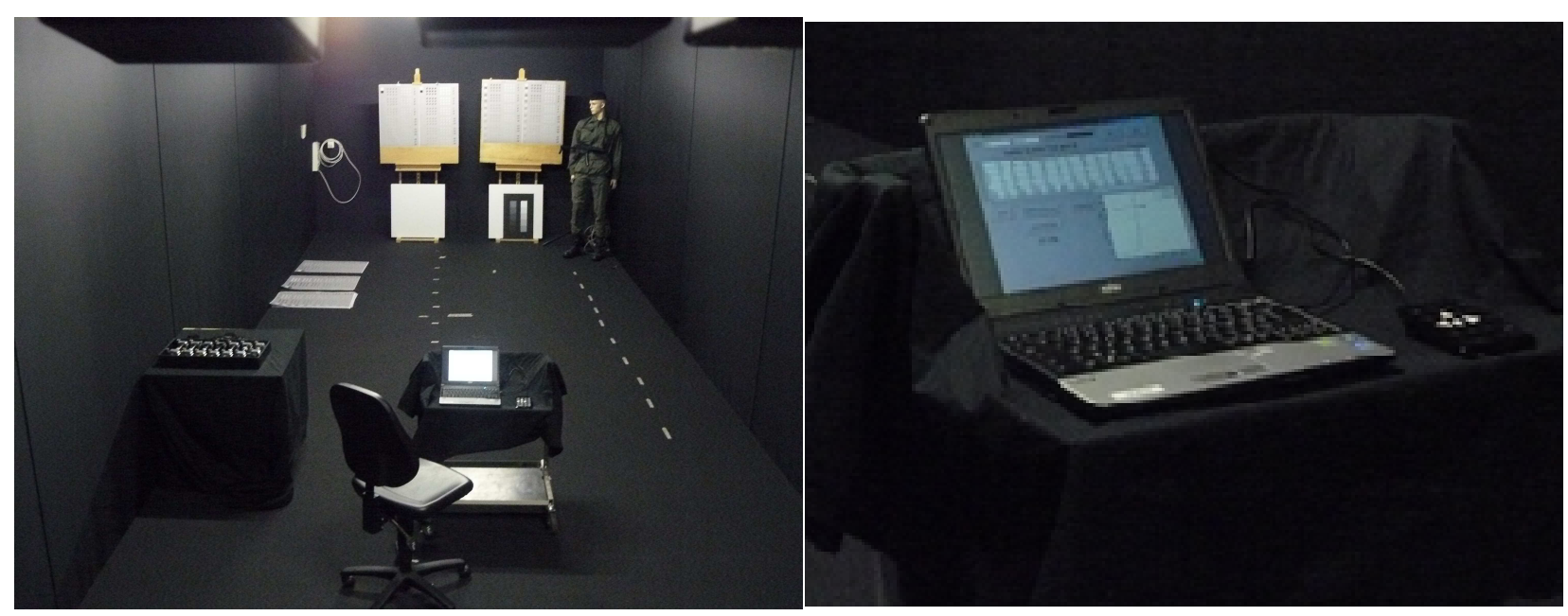

Figure 1. Overview of the test setup : on the left side: general view of the test darkroom On the right side: laptop with the keypress software running. The keypad is visible.

\subsection{TOD test charts and Data Collection}

According to the TOD guidelines ${ }^{1}$, the test consists of Visual Acuity (VA) and a Contrast Sensitivity (CS) measurements. Night vision VA and CS test charts were manufactured based on the test charts described by Hogervorst et al. ${ }^{6}$. See Figure 1. Visual Acuity charts consist of 18 lines with 8 triangle test patterns of fixed contrast and decreasing triangle size; Contrast Sensitivity charts consist of 18 lines with 8 test patterns of fixed triangle size and decreasing contrast.

The VA charts are available in two types starting with different triangle size, each of them printed in three versions with different triangle orientations in order to avoid bias of knowing a chart by-heart. From the biggest triangle, at each step the size is decreased by $0.06 \log$ units (i.e. $15 \%$ in size) and each step is made of four dark triangles (for negative contrast) and four white triangles (for positive contrast).

The CS charts are available for nine triangles sizes. Three versions exist for each target size with different triangles orientations in order to avoid sequences learning by the testers. Target contrast on the charts is decreased from highest to lowest contrast in $0.10 \log$ unit steps (i.e. $25 \%$ in contrast).

Contrast is defined as $C=\frac{L_{t}-L_{b}}{L_{b}}$,

where $\mathrm{L}_{\mathrm{t}}=$ the luminance of the test target, and $\mathrm{L}_{\mathrm{b}}$ the luminance of the background.

Note: special care needs to be taken to correctly characterize the test charts for $\mathrm{I}^{2}$ devices as they are sensitive in the Near IR region, outside the visual region for which photopic contrast has been defined. For details with respect to the test chart reflective properties and the resulting test pattern contrasts in the visual and Near IR region we refer to the Appendix. 


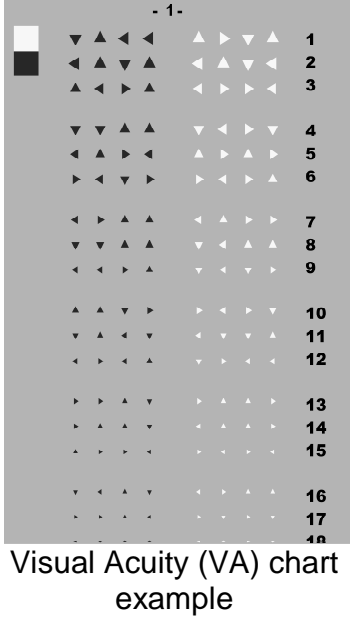

Figure 2. Examples of the TOD test charts produced for the test campaign

\subsection{Observer tests}

\subsubsection{Observer task}

The task in the TOD test is to judge the orientation (Apex Up, Down, Left or Right) of the triangles on the test chart. It is mandatory to respond to all selected triangles, even if one is unsure. This so-called forced-choice procedure eliminates observer bias.

For the conventional $\mathrm{I}^{2}$ tubes, the observers were seated on a chair, looking through the monocular and holding the monoculars in one hand. A keyboard was used by the other hand to record the TOD responses. Small systematic movement of the sight allowed the observers to sort out the interesting detail from the image noise.

The experiments with the ICMOS camera were performed offline on recorded material. During the recordings, the ICMOS camera was placed on rail with a linear translation stage. Frames were grabbed during a defined translation for avoiding any aliasing. Contrast enhancement by histogram stretching was fixed and identical for all the observers. Images were displayed on a LCD Ilyama 24" Prolite E2407HDSD Screen at a frame rate of 50 images per second. A test session covered one device tested at a given night and took about 1 hour.

The observers responses were collected by a keypress on a Photonis GUI installed on a laptop. Its screen was hidden to avoid parasite light to interfere with the test. The observers were asked to proceed with the chart one line after the other, pressing the key corresponding to the orientation 'up', 'down','right','left' he thought to resolve. As the display was hidden, a sound signal indicated the observer that he did not miss a triangle. The test was proceeded until all 18 lines were read or until the triangle discrimination success rate was around $25 \%$.

\subsubsection{Test order}

For each observer and each device, first all VA measurements were carried out. The test was performed at the five night levels indicated in Table 2. For the darkest scenario, at level 4 and 5, the test was realized at a distance of $3 \mathrm{~m}$ and started with the VA charts with the largest triangle size. At night level 1,2 and 3, the observers were located five meters away from the target. At level 1 and 2, the format of the VA chart was changed to start at a lower triangle size, to take into account the higher limiting resolution reached in such type of situation. Sensor Visual Acuity (VA) is defined as the reciprocal size threshold achieved by the equipment on the VA Chart, and is expressed in reciprocal angular units (mrad $\left.{ }^{1}\right)$.

From these data, VA thresholds for all light levels for negative and positive contrast test patterns were calculated using the analysis procedure described in section 2.5. These thresholds serve as a baseline for the triangle sizes at which the CS measurements need to be performed.

Next, all CS measurements were carried out, starting from the largest triangle size available down to the negative/positive average threshold estimated SA at the VA step. From these measurements, CS (or the reciprocal, contrast threshold) was calculated for negative and positive test patterns at all sizes at all five levels using the procedure described in section 2.5 


\subsubsection{Observers}

The test group was made of four male observers: AL, HM, LC and SG, respectively 28, 46, 42 and 38 years old. All of them were tested and had normal or corrected to normal visual acuity. They were all experienced in using night vision devices.

\subsection{Data analysis}

The collected data consists of a relationship between success rate and stimulus strength (triangle reciprocal size or contrast) for positive and negative test patterns for all devices at five night levels, i.e. for $2 * 4 * 5=40$ conditions and for one VA test and a series of CS tests According to the TOD guidelines ${ }^{1}$, these data are fitted against a Weibull cumulative function by maximizing the likelihood ratio. The validity of the fit is statistically tested with a $\chi^{2}$ test $t^{2}$. The stimulus threshold is computed from the Weibull fit and corresponds to the $75 \%$ success rate. Figure 3 shows an example condition with separate Weibull fits for positive and negative contrast targets. The weighted log average over the observers is taken as the threshold value. Next, for each condition the curve of the log contrast threshold vs. reciprocal triangle size (in $\mathrm{mrad}^{-1}$ ) is least-square fitted against the polynomial of the lowest order that sufficiently describes the data according to a $F$-test ${ }^{1}$. Because the error in VA is in the size domain while the errors of the other thresholds are in the contrast domain, the VA error is converted into a contrast error by multiplying this value by the estimated slope in the high reciprocal triangle size region. The results are TOD curves and are presented in the graphs in Section 3.

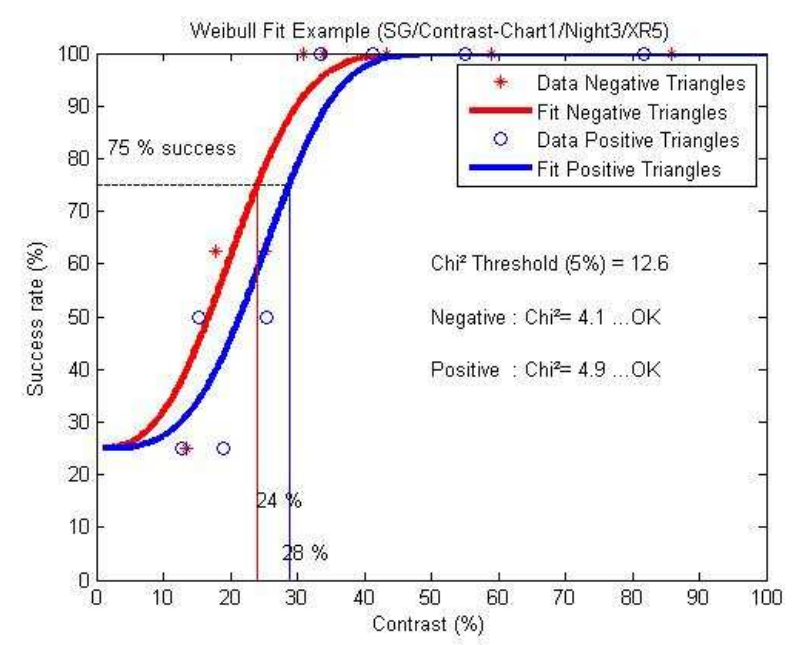

Figure 3. Weibull psychometric function fit example for a contrast threshold measurement with positive and negative targets.

\section{RESULTS}

\subsection{Performance per device}

In this section, the different results obtained with the four different devices are presented. The curves are plotted for the five night levels enumerated in section 2.3, except for the ICMOS which is not sensitive enough to perform a TOD test in the darkest situation (night level 5).

Figure 4 shows the results for the $\mathrm{XR} 5^{\mathrm{TM}}$ (left graph) and $\mathrm{XD} 4^{\mathrm{TM}}$ (right graph), while Figure 5 provides the results for the Supergen ${ }^{\circledR}$ (left graph) and the ICMOS (right graph). Most curves are well-described by a linear relationship. The curves roughly shift in vertical direction when the light level decreases.

The curves are plotted at each contrast polarity separately, emphasizing a shift between negative (plain curves) and positive (dotted curves) stimuli. Thresholds are lowest for negative contrasts. This effect is particular present for the Image Intensifiers and is less visible for the ICMOS, possibly because the digital images were contrast enhanced before being presentated to the observers. 

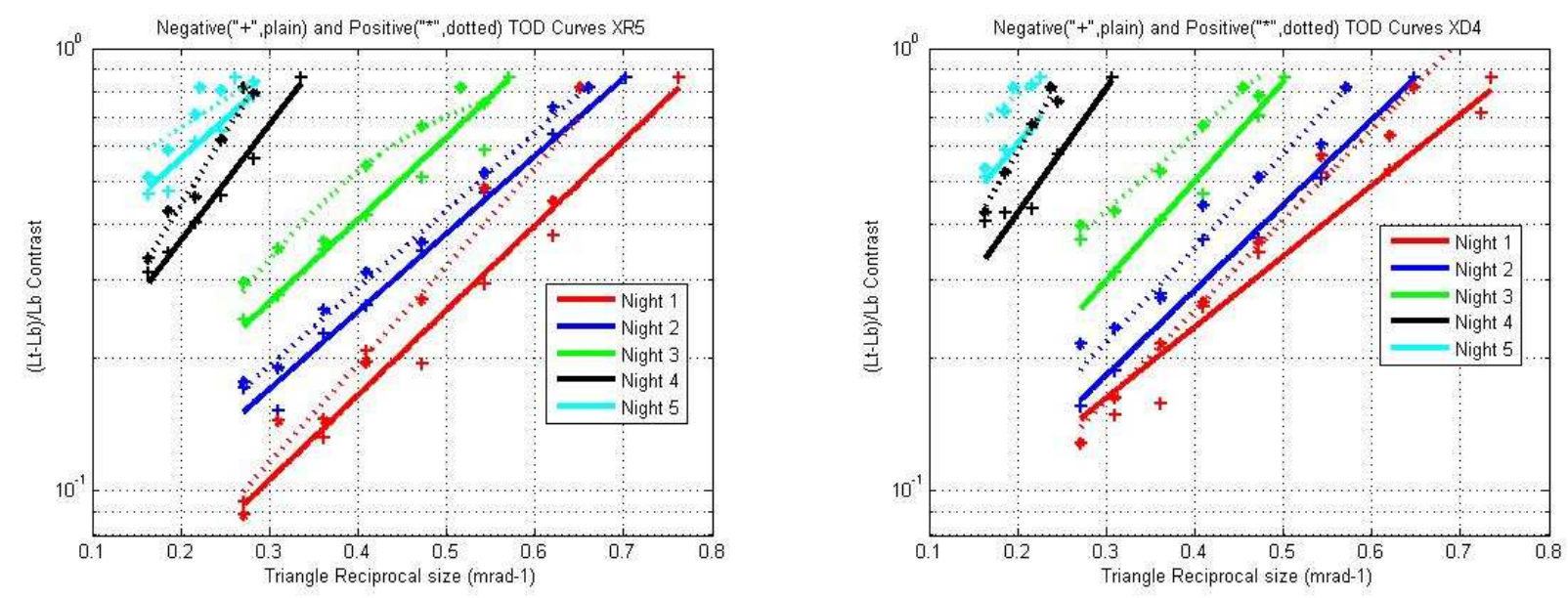

Figure 4. Performance of monocular integrated with XR5TM (left) and XD4TM (right) monocular in night levels 1 to 5.
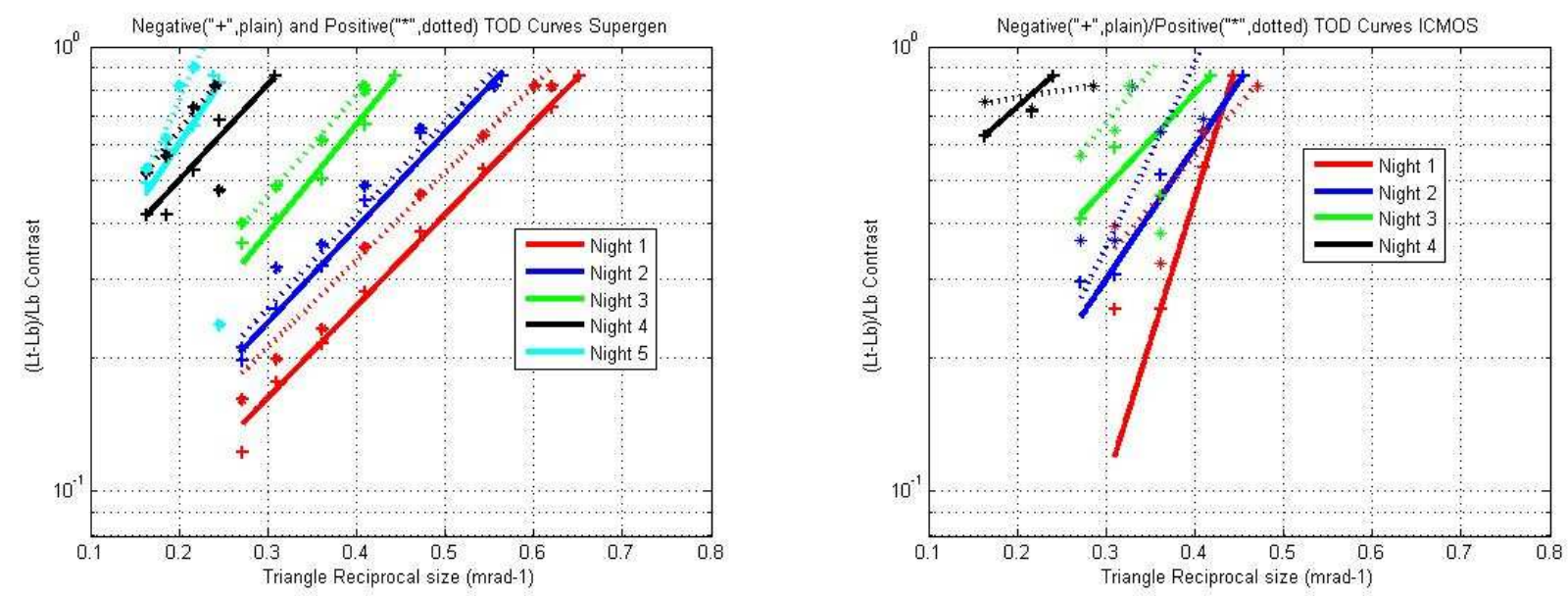

Figure 5. TOD Curve of the monocular integrated with Supergen monocular (left) in night level 1 to 5 and for the ICMOS camera (right) in night 1 to 4 .

\subsection{Comments on results: difference between devices per night level}

All results are discussed on negative contrasts as remarks done between devices remain valid for positive contrasts. Comparison between negative and positive contrasts are available on Figure 4 and 5 above.

\section{Night level 1}

At the highest night level, performance is expected to be resolution limited; this fact is verified on the TOD Curves (see Figure 6)

- $\quad \mathrm{XD} 4^{\mathrm{TM}}$ and $\mathrm{XR} 5^{\mathrm{TM}}$ have a very similar limiting resolution (respectively 64 and $66 \mathrm{lp} / \mathrm{mm}$ ), their curve meet while approaching the sensor visual acuity (VA).

- The Supergen is clearly lagging behind this two tubes: its VA on the negative chart is equal to $0.65 \mathrm{mrad}^{-1}$ that is to say $15 \%$ less than XD4 ${ }^{\mathrm{TM}}$ and $\mathrm{XR} 5^{\mathrm{TM}}$.

- The chosen ICMOS reaches lower VA performance: its value is 0.44 and $0.47 \mathrm{mrad}^{-1}$. 


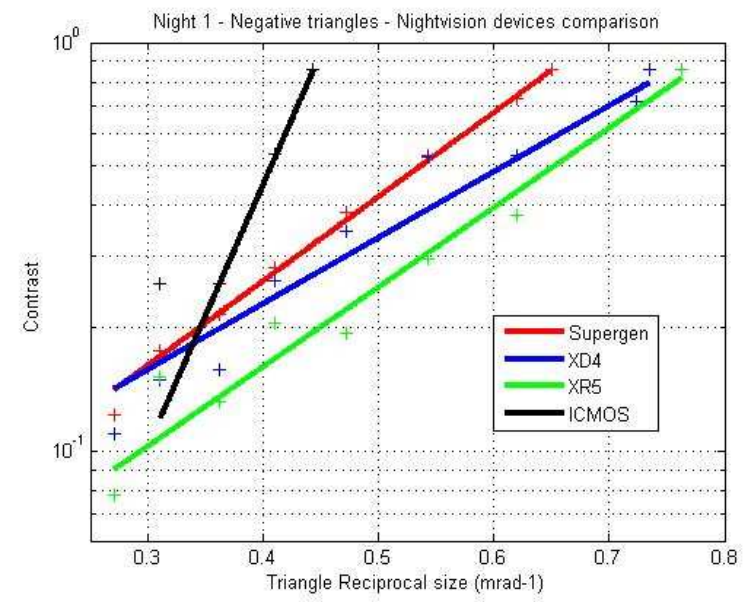

Figure 6. Performance of XR5TM, XD4TM, SuperGen® and ICMOS at Night level 1.

\section{Night level 2 and 3}

Although night level 2 is still relatively high, sensor performance at this level is significantly lower than at night level 1 (see Figure 7).

- $\quad$ For standard $\mathrm{I}^{2}$ monocular, the drop in performance with illuminance level is highest for the best performing tube:. To illustrate this, for negative VA chart, the fall is $20 \%$ for the Supergen ${ }^{\circledR}(\mathrm{SNR}=18), 13 \%$ for the $\mathrm{XD} 4^{\mathrm{TM}}(\mathrm{SNR}=20)$ and $7 \%$ for the $\mathrm{XR} 5^{\mathrm{TM}}(\mathrm{SNR}=25)$.

- $\quad$ The Supergen is firmly staying behind the XD4 ${ }^{\mathrm{TM}}$, at both low/high contrast discrimination task, the difference between $\mathrm{XD} 4^{\mathrm{TM}}$ and $\mathrm{XR} 5^{\mathrm{TM}}$ are mostly apparant at the highest triangle reciprocal size.

- $\quad$ ICMOS VA for the negative test pattern is comparable to acuity at the highest night level; contrast sensitivity however decreases. VA for the negative test pattern decreases by $15 \%$.

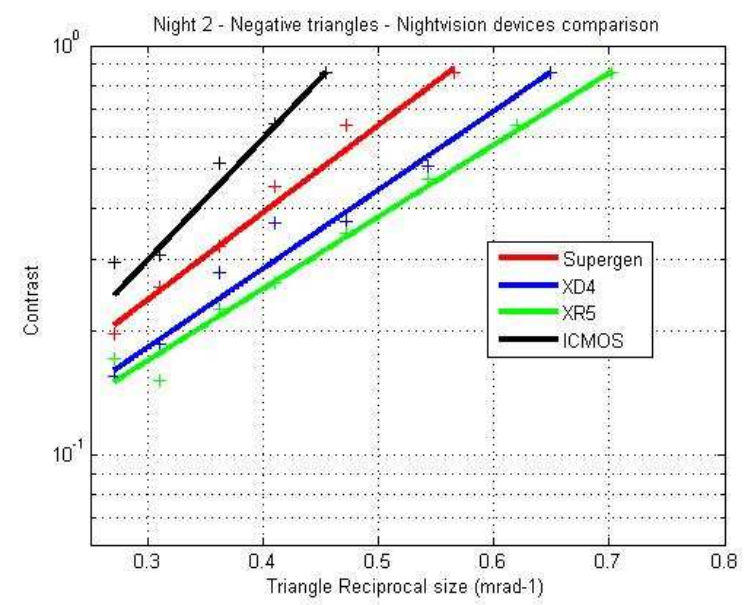

Figure 7. Performance of XR5TM, XD4TM, SuperGen® and ICMOS at Night level 2.

At Night Level 3, the conclusions made on night level 2 are essentially remaining (see Figure 8):

- Conventional $\mathrm{I}^{2}$ are further losing VA performance: $-33 \%$ for SuperGen ${ }^{\circledR}$ and $\mathrm{XD} 4^{\mathrm{TM}}$ and $-13 \%$ for $\mathrm{XR} 5^{\mathrm{TM}}$. This suggests the impact of noise on VA. As a result, tubes with the best SNR have an advantage in resolving small details in a darker environment.

- $\quad$ Supergen ${ }^{\circledR}$ is dropping out and clearly achieves far less performance than $\mathrm{XD} 4^{\mathrm{TM}} / \mathrm{XR} 5^{\mathrm{TM}}$.

- The ICMOS is behaving in a similar way as at the two previous night levels: its VA performance is not changed very much and the gap with the conventionnal tubes decreases. Still, the system lacks capacity to discriminate low contrast targets. 


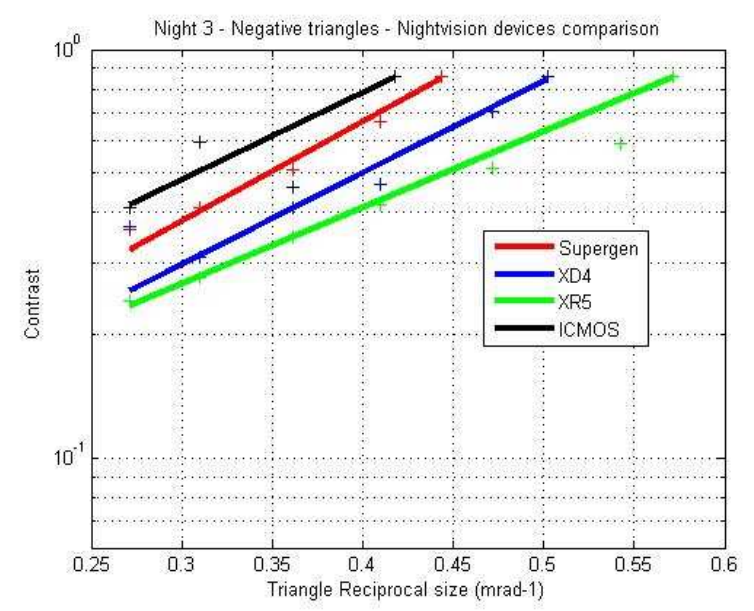

Figure 8. Performance of XR5TM, XD4TM, SuperGen® and ICMOS at night level 3.

\section{Night level 4 \& 5}

At Night Level 4, Figure 9 (left side) leads to the following observations:

- $\quad \mathrm{XR}^{\mathrm{TM}}$ still shows an advantage to discriminate smal details or low contrast targets

- Conventionnal $\mathrm{I}^{2}$ are losing performance $-43 \%$ for $\mathrm{XR}^{\mathrm{TM}},-40 \%$ for $\mathrm{XD} 4^{\mathrm{TM}},-21 \%$ for Supergen ${ }^{\circledR}$. It is remarkable that Supergen ${ }^{\circledR}$ and $\mathrm{XD} 4^{\mathrm{TM}}$ show equal VA. This may be explained by the contrast and the temporal noise density, killing the MTF difference at a reciprocal triangle size close to up to $0.3 \mathrm{mrad}^{-1}$.
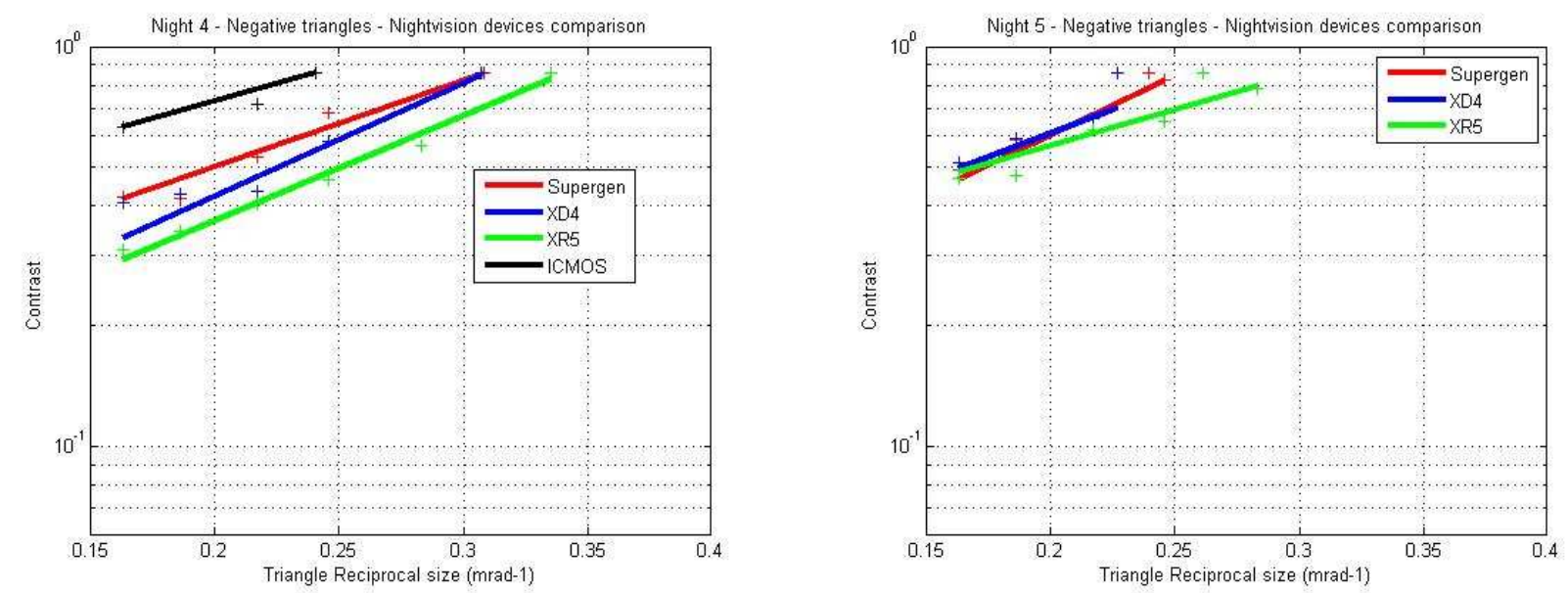

Figure 9. Performances of XR5TM, XD4TM, SuperGen® and ICMOS in night levels 4 and 5.

At Night Level 5, Figure 9 (right side) leads to the following observations:

- At Night Level 5, XR5 $5^{\mathrm{TM}}$ still clearly shows VA advantage by only loosing $16 \%$ VA performance from Night Level 4 to Night Level 5

- At Night Level 5, other $\mathrm{I}^{2}$ (both $\mathrm{XD} 4^{\mathrm{TM}}$ and Supergen ${ }^{\circledR}$ ) do not reveal any difference stating likely the hypothesis that the most important limiting factor is the low output luminance and that SNR difference is no more revealed by the test. Also the difference that may occur at the low frequency MTF is also not shown (same limiting contrast)

\section{DISCUSSION - ANALYSIS}

\subsection{Relation between $\mathrm{I}^{2}$ performance and TOD curves}

As we defined at section 1, SNR and LR being the main parameters for defining the performance level, we expect a relationship between the intrinsic $\mathrm{I}^{2}$ performance and the TOD hierarchy. Moreover, it is mostly interesting to pin out what part of the TOD curve in which illumination is either influenced by MTF/Resolution or by SNR. 
The ability to discriminate small triangles is expected to be linked to the LR/MTF performance: the higher the LR/MTF, the smaller the triangles that can be distinguished, and thus the higher VA. In addition, the ability to distinguish low contrast targets is linked to noise level: the higher the SNR, the lower the expected contrast at which observer will be able to discriminate triangles. In this way, exercice is to give an horizontal shift on TOD curves in the ratio of VA/LR (VA/LR expected to be stable as higher VA is provided by higher LR) and a vertical shift in the ratio of Contrast $\mathrm{x}$ SNR (Contrast x SNR expected to be stable as lower contrast threshold is provided by higher SNR). In this way, if TOD performances is directly linked to $\mathrm{I}^{2}$ performances, all curves shall be superimposed.

Figure 10 shows the results of the TOD curves conversion for 3 main Night Levels for monocular integrating XR5 ${ }^{\mathrm{TM}}$, $\mathrm{XD} 4^{\mathrm{TM}}$ and SuperGen®.
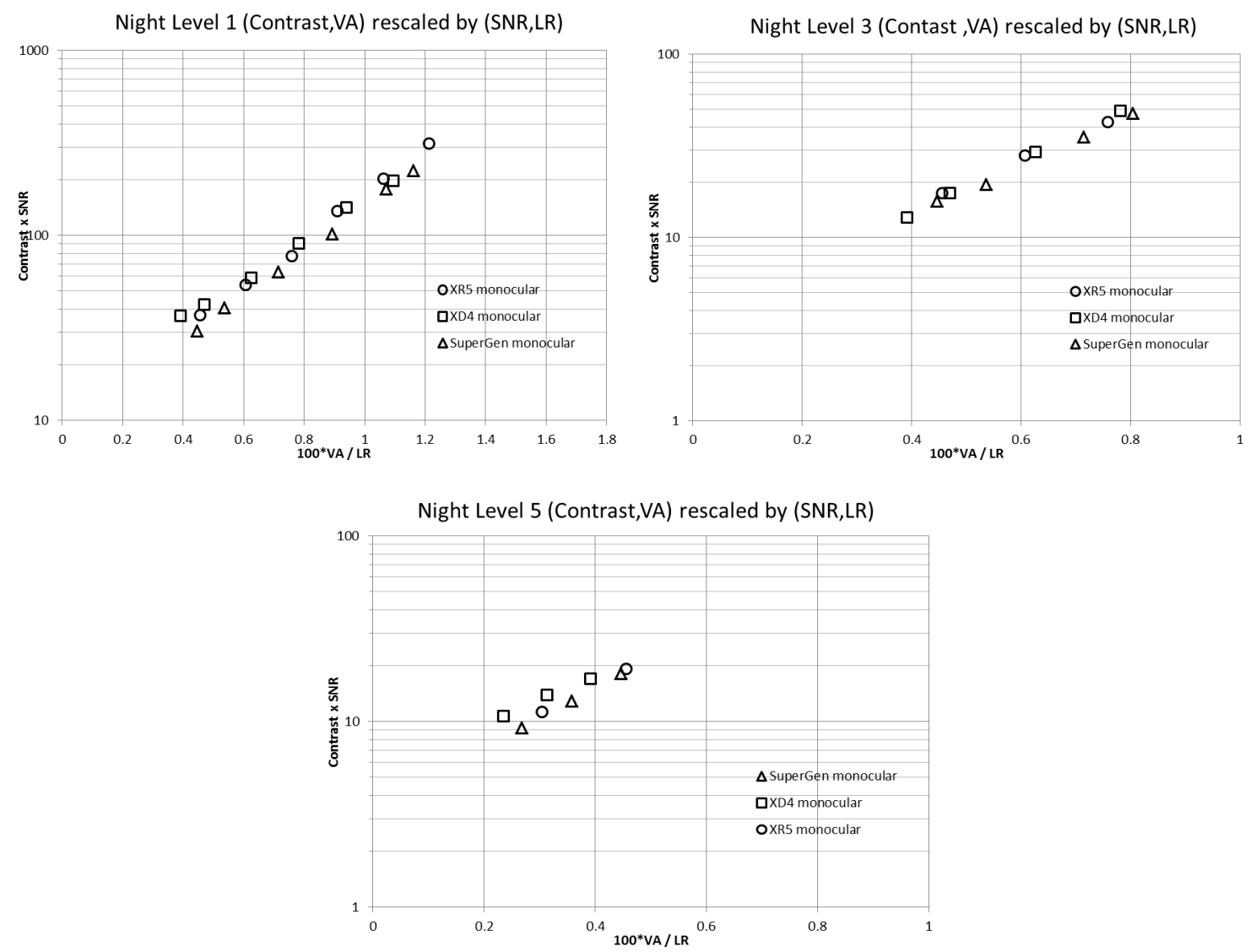

Figure 10. Rescaled TOD curves for I2 monoculars for Night levels 1, 3 and 5.

It is noticeable that curves of the 3 performances levels (SuperGen ${ }^{\circledR}$ to $\mathrm{XR} 5^{\mathrm{TM}}$ ) related to Night 1 and Night 3 conditions enhance a good correspondance. That anticipates the fact that range will be strongly linked to the $\mathrm{I}^{2}$ performances. It also again illustrates the accuracy of the psychophysical measurement of the TOD data.

At Night level 5 the correspondence is less strong: we can see a systematic effect of $\mathrm{I}^{2}$ type here. This effect may be ascribed to the low display luminance: Night level 5 corresponds to 70 lux on the $\mathrm{I}^{2}$ photocathode corresponding to less than $1 \mathrm{~cd} / \mathrm{m}^{2}$ at the output. At this brightness level, performance is also limited by the human eye.

\subsection{Contrast polarity results / Contrast definition}

Looking at the measurement results, negative and positive contrasts show different TOD thresholds for the $\mathrm{I}^{2}$ tubes at the lowest light levels (Night Level 4 and Night Level 5) and at any light level for ICMOS. Visual Acuity is higher for negative contrast test patterns which is directly confirmed by the feeling reported by the observers. This result is however difficult to interpret from sensor point of view because one would expect to better resolve white triangle with a greater SNR onto grey background than a dark triangle (low SNR) onto the same background.

During the study the contrast definition has also been discussed. In the TOD guidelines ${ }^{1}$ the Weber contrast is used. An alternative definition is Michelson's contrast: :

$$
C=\frac{L_{t}-L_{b}}{L_{b}} \text { (Weber contrast définition) } \quad C=\frac{L_{t}-L_{b}}{L t+L_{b}} \text { (Michelson contrast definition) }
$$


where Lt and Lb stand repectively for Luminance of the triangle and for Luminance of the background.

Weber contrast is the most appropriate to express the visual perception of a defined target on a background level but the Michelson contrast is often used by $\mathrm{I}^{2}$ community when dealing with Modulation Transfer Function (MTF). The MTF curve which is a crucial parameter for any imaging device, quantifies the modulation contrast degradation. This parameter is handled with Michelson contrast as for object contrast. So the question raised during the study, what is the impact of those two definition when inserting these in the TOD results. The following curves give an example:
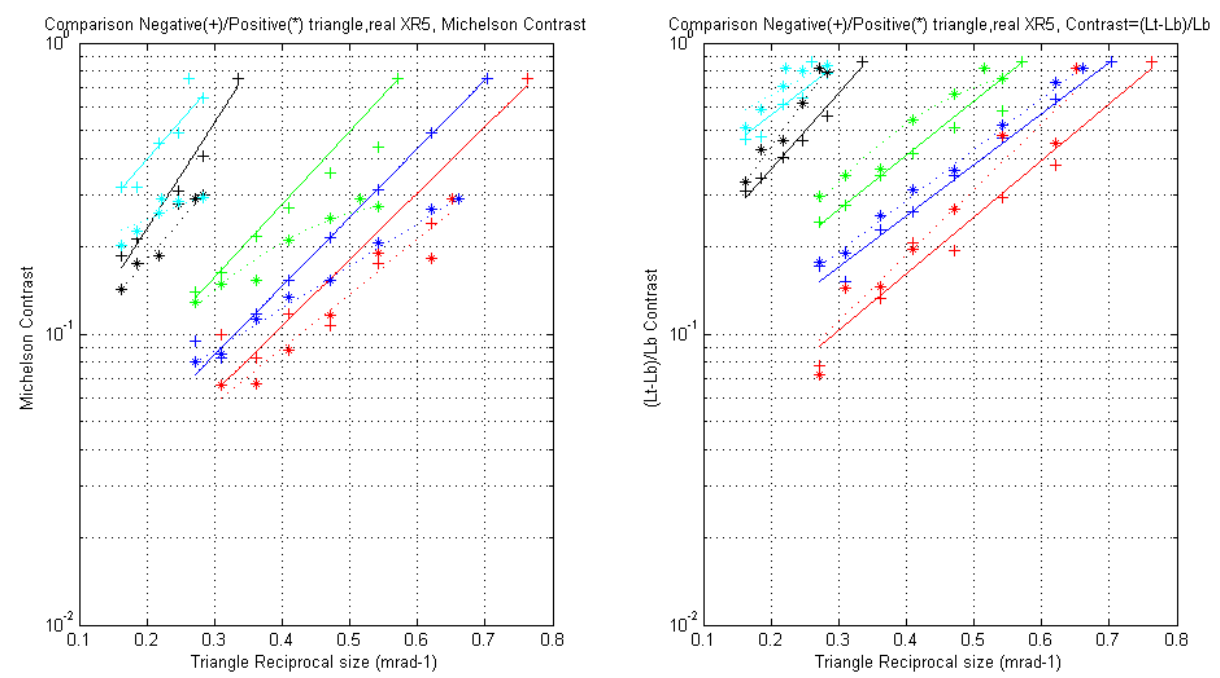

Figure 11. Michelson vs Weber contrast comparison for XR5TM and Negative vs Positive contrasts

A $100 \%$ Weber contrast corresponds to a triangle twice more luminous than the background, it corresponds to a much lower Michelson contrast droping down to 30\%. The curve lies automatically lower as Michelson ratio is by definition lower than Weber. As a surprise, one can see that the positive are better resolved which is the opposite conclusion to the other contrast definition. In addition, Michelson contrast gives a larger difference between positive and negative but as for Weber contrast, the two contrast polarity curves seem to meet at the low contrast/low reciprocal triangle size. In this case $(\mathrm{Lt}<<\mathrm{Lb})$, both definition are consistent except a factor 2.

\subsection{Range prediction examples}

From the TOD target acquisition model ${ }^{7}$, field range can be predicted from the TOD curve as a function of the target size, the task level required (Detection, Recognition or Identification of the target) at a given probability, the visibility through the atmosphere and the illumination level. Figure 12 shows an example:

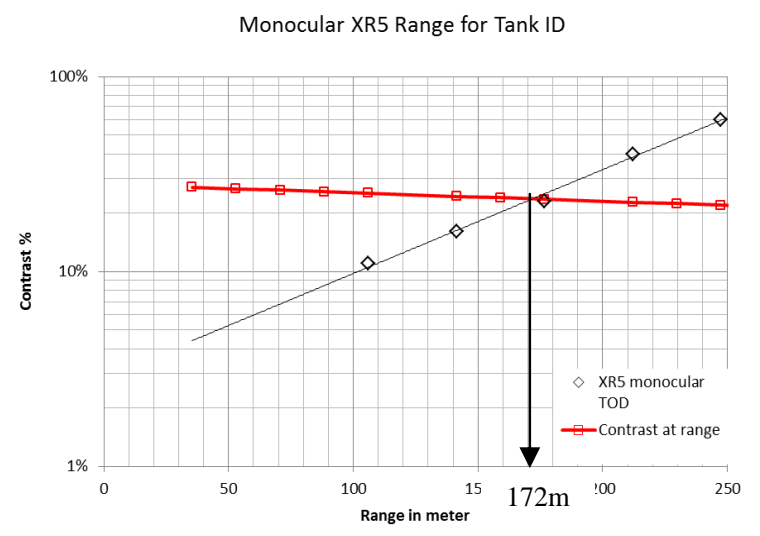

Figure 12. Tank ID Range prediction example for a monocular at Night 1

Range can be deduced as the intersection point between target acquisition results derived from TOD curves and the target contrast at range. Other example can be showed hereby using the same protocol:

Table 3 : Tank ID range examples 


\begin{tabular}{|l|c|c|c|c|c|c|}
\hline Task & \multicolumn{2}{|c|}{ Tank ID at Night Level 2 (15mlux on target) } & \multicolumn{3}{|c|}{ Tank ID at Night Level 4 (0.9 mlux on target) } \\
\hline Equipement & $\begin{array}{c}\text { Monocular } \\
\text { XR5 }^{\mathrm{TM}}\end{array}$ & $\begin{array}{c}\text { Monocular } \\
\text { XR4 }^{\mathrm{TM}}\end{array}$ & $\begin{array}{c}\text { Monocular } \\
\text { SuperGen }{ }^{\circledR}\end{array}$ & $\begin{array}{c}\text { Monocular } \\
\text { XR5 }^{\mathrm{TM}}\end{array}$ & $\begin{array}{c}\text { Monocular } \\
\text { XD4 }^{\mathrm{TM}}\end{array}$ & $\begin{array}{c}\text { Monocular } \\
\text { SuperGen }{ }^{\circledR}\end{array}$ \\
\hline Eval. Range (m) & 140 & 130 & 110 & 52 & 48 & 30 \\
\hline
\end{tabular}

With the following atmosphere and target acquisition parameters:

- Visibility $=1000 \mathrm{~m}$ (just out of fog condition)

- Target contrast $~ 30 \%$ (chosen arbitrary)

- $\quad$ Target set and task $=$ Tank ID (Identification) at Probability 0.75

- $\quad$ Characteristic dimension ${ }^{8}=3.11 \mathrm{~m}$

- $\quad$ TOD magnifying factor $\mathrm{M} 75^{9,10}=8.8$

Table 4 : Human activity (weapon / non weapon held) discrimination range examples

\begin{tabular}{|l|c|c|c|c|c|c|}
\hline Task & \multicolumn{3}{|c|}{ Tank ID at Night Level 1 (100mlux on target) } & \multicolumn{3}{|c|}{ Tank ID at Night Level 3 (4.3 mlux on target) } \\
\hline Equipement & $\begin{array}{c}\text { Monocular } \\
\text { XR5 }^{\mathrm{TM}}\end{array}$ & $\begin{array}{c}\text { Monocular } \\
\text { XD4 }^{\mathrm{TM}}\end{array}$ & $\begin{array}{c}\text { Monocular } \\
\text { SuperGen }{ }^{\circledR}\end{array}$ & $\begin{array}{c}\text { Monocular } \\
\text { XR5 }^{\mathrm{TM}}\end{array}$ & $\begin{array}{c}\text { Monocular } \\
\text { XD4 }^{\mathrm{TM}}\end{array}$ & $\begin{array}{c}\text { Monocular } \\
\text { SuperGen } \AA\end{array}$ \\
\hline Eval.Range (m) & 52 & 47 & 41 & 33 & 30 & 25 \\
\hline
\end{tabular}

With the following atmosphere and target acquisition parameters:

- $\quad$ Visibility $=1000 \mathrm{~m}$ (just out of fog condition)

- $\quad$ Target contrast $~ 30 \%$ (chosen arbitrary)

- $\quad$ Target set and task $=$ Weapon $/$ non Weapon discrimination at Probability 0.75

- $\quad$ Characteristic dimension $12=0.25 \mathrm{~m}$

- $\quad$ TOD magnifying factor $M 75^{9,10}=2.4$

\subsection{Future progress}

The next step in our research will be to extensively test $\mathrm{I}^{2}$ with higher performance, with different settings (Gain, Maximum Output brightness) and other design characteristics that one would expect to influence the observer task. For instance, we are aiming at giving a precise figure on the cognitive advantage of the black and white phosphor (P45) that Photonis developed on the recent Onyx $\mathrm{I}^{2}$.

In order to use the TOD protocol in a systematic and efficient way, Photonis is investigating a solution whose principle is to dynamically project spectral images and/or movies on display with a preset spectral distribution. Ideally we would target to project the targets with a spectrum representative for the night spectrum of the 5 levels from visible to short wave infrared. The system would be able to project at a fixed location on the screen all triangle series required to draw the TOD curve. The generation of the sequence could be adaptative.

Finally, Photonis will use this extended results database to continue and to complete the work done about the range prediction modelling as a tool for development choice and benchmarking investigation.

\section{CONCLUSION}

Thanks to the collaborative work done with TNO institute, Photonis shows in this paper that all mandatory means and protocol are used to accurately apply the proven concept of the Triangle Discrimination Orientation test method. A first set of standard $\mathrm{I}^{2}$ and an ICMOS have been characterized and the test results led to complete and reliable results for the main night level conditions. A good prediction of the range performance can then be derived and it is expected to apply the same exercice for any other devices. Photonis is constantely focused on customer expectations, and the purpose of the paper was to demonstrate that the company is able to support integrators and end users by providing test and modelling tools (test means, method and range calculation) for field performances evaluation of night vision equipements. 


\section{ACKNOWLEDGMENTS}

The authors wish to thank the observers for participation in this measurement campaign.

\section{REFERENCES}

[1] Bijl, P.\& Valeton, J.M. (1999). Guidelines for accurate TOD measurement. SPIE Proceedings, Vol. 370114 25.

[2] P. E. Greenwood et M. S. Nikulin, A Guide to Chi-Squared Testing, John Wiley and Sons, 1996.

[3] STANAG 4350

[4] MIL-STD-150A

[5] Bijl, P.\& Valeton, J.M. (1998). TOD, the alternative to MRTD and MRC. Optical Engineering 37, 7,1976 1983.

[6] Hogervorst, M.A. \& Bijl, P. (2003). Testkaarten voor meting van resolutie en contrastgevoeligheid: een gebruikershandleiding (Report TM-03-A005) Soesterberg, The Netherlands: TNO Human Factors (in Dutch).

[7] Bijl, P. \& Vries, S.C. de, (2010). Visual Acuity, Contrast Sensitivity and Range Performance with compressed motion video, Optical Engineering, Vol. 49, 103203, doi:10.1117/1.3503950.

[8] Vollmerhausen, R.H., Jacobs, E., Hixson, J. \& Friedman, M. (2006). The Targeting Task Performance (TTP) Metric: A New Model for Predicting Target Acquisition Performance. Technical Report AMSEL-NV-TR-230, NVESD, Fort Belvoir, VA22060 (January 2006).

[9] Bijl, P., Reynolds, J.P., Vos, W., Hogervorst, M.A. \& Fanning, J.D. (2011). TOD to TTP calibration. In: Infrared Imaging Systems: Design, Analysis, Modeling, and Testing XXII, 8014, 80140L; doi:10.1117/12.887219.

[10]Bijl, P., Hogervorst, M.A., Vos, W., "Direct comparison of TOD, TTP, and tactical vehicle identification performance data underlying the TTP metric." (Report TNO-DV 2011 C486). Soesterberg, The Netherlands: TNO Behavioural and Societal Sciences, (2012).

[11] Moyer, S., Hixson, J. G., Edwards T. C., Krapels K., "Probability of identification of small hand-held objects for electro-optic forward-looking infrared systems," Opt. Eng. 45, 063201 (2006).

[12] Beintema, J.A., Bijl, P., Hogervorst, M.A., Dijk, J. "Target Acquisition performance: effects of target aspect angle, dynamic imaging and signal processing," Infrared Imaging Systems: Design, Analysis, Modeling, and Testing XIX, $6941169410 \mathrm{C}$ (2008).

\section{Appendix: TOD test chart details}

Charts were printed by an external professional printer company located in Brive, France, using pure B\&W inkjet printer, on A1 isoformat. First, grey level pantone graphs were printed. This was used to match the actual luminance curve with the digital grey level prescribed in the provided file. Charts were then generated using this calibration curve, in such a way that the grey level background presents luminance in the middle of the darkest and the whitest stimulus present on chart to yield $+/-90 \%$ contrast on the VA charts.

Adopted convention is $\mathrm{C}=\left(\mathrm{L}_{\mathrm{T}}-\mathrm{L}_{\mathrm{B}}\right) / \mathrm{L}_{\mathrm{T}}$ with $\mathrm{L}_{\mathrm{T}}, \mathrm{L}_{\mathrm{B}}$ representing the luminance of the triangle and of the background, respectively.

Photopic contrasts were measured with a Minolta Luminance meter (LS100 model) through a photopic filter.

The high sensitivity of an $\mathrm{I}^{2}$ device in the Near IR requires the characterization of the chart spectral reflectivity at both the visual wavelengths (from $4000-7000 \mathrm{~nm}$ ) and the Near IR wavelengths (from $700-1100 \mathrm{~nm}$ ). The spectral reflectivity of the Charts with different ink densities was measured with a spectrometer (Filmetrics F20 analyser coupled to Hamamatsu UV-VIS fiber Light source), The results are shown in Figure 13. The measurement shows that the spectral behavior in the NIR is not dramatically different from the visual part. Nevertheless, the spectral reflectivity of the paper is not perfectly flat and exhibits an excess in the low wavelength which will affect the contrast integrated over the spectral range of the $\mathrm{I}^{2}$. 


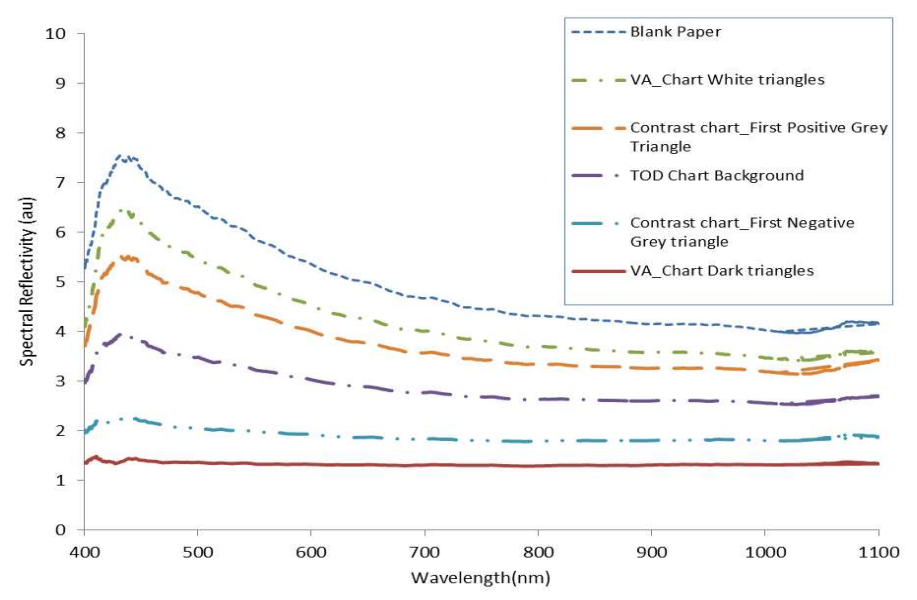

Figure 13. Chart and ink spectral response (arbitrary unit)

Figure 14 shows the spectral response from Figure 13, weighted with the spectral sensitivity of the sensor and the emission of the light source (black body at 2856K), Fom these curves we can deduce the actual contrasts as seen throught the I 2 devices. Examples are provided in Table 5. As we can see from this Table, spectrally integrated contrast is about 0.6 times lower than the photopic contrasts, and this fraction is constant across the reflection levels.

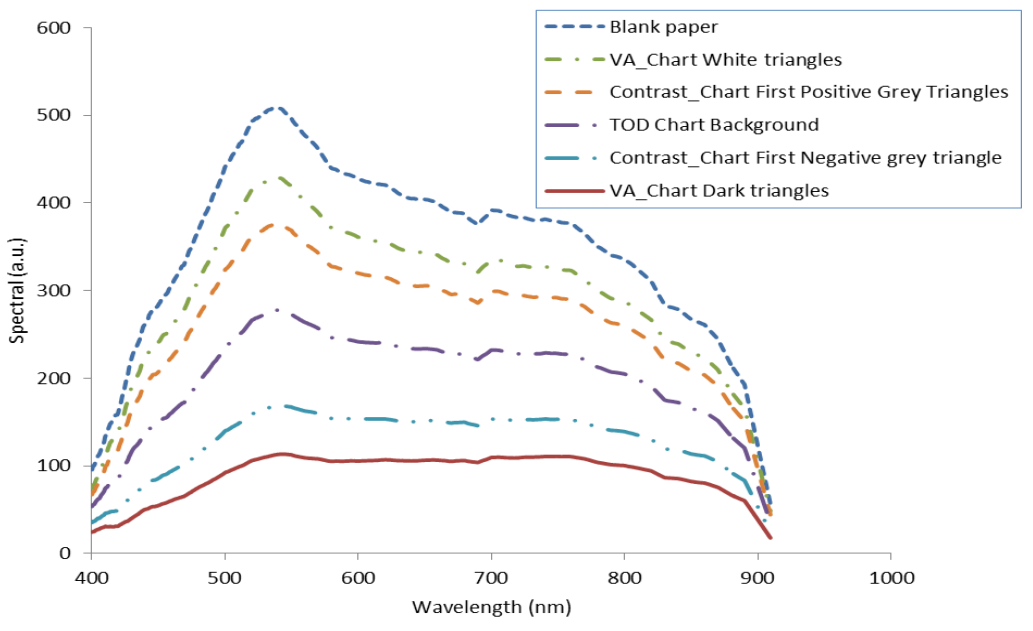

Figure 14. Integrated Spectral Response of the Charts

Table 5: photopic and spectrally integrated contrasts of the test targets on the chart

\begin{tabular}{|l|c|c|}
\hline Stimulus & Photopic Contrasts & $\begin{array}{c}\text { Spectrally Integrated } \\
\text { Contrast }\end{array}$ \\
\hline VA negative triangle & $-86 \%$ & $-55 \%$ \\
\hline VA positive triangle & $82 \%$ & $48 \%$ \\
\hline First Negative Contrasts on Contrasts Chart & $-59 \%$ & $-36 \%$ \\
\hline First Positive Contrasts on Contrasts Chart & $55 \%$ & $31 \%$ \\
\hline
\end{tabular}

